\title{
Régulation paracrine des fonctions leydigiennes
}

\author{
Hervé LeJeune \& José Maria Saez.* \\ INSERM U.307, Hôpital Debrousse, 29 rue Soeur Bouvier, 69322 Lyon Cedex 05.
}

\section{INTRODUCTION}

Le testicule a deux fonctions complémentaires : d'une part une fonction endocrine, correspondant essentiellement à la production des stéroïdes sexuels masculins, les androgènes et d'autre part une fonction exocrine, la production des gamètes mâles, les spermatozoïdes.

L'architecture tissulaire du testicule rend compte de ces 2 fonctions; le testicule comporte en effet deux compartiments fonctionnels, $1^{\circ}$ - le tissu interstitiel, conjonctif, vascularisé, contenant les cellules de Leydig responsables de la fonction endocrine et $2^{\circ}$ - les tubes séminifères, de nature épithéliale, avasculaires, contenant les cellules germinales et les cellules de Sertoli qui servent de support à la spermatogénèse. La séparation entre ces deux compartiments, appelée barrière hémotesticulaire, est constituée successivement des cellules péritubulaires myoïdes, de la membrane basale propre du tube séminifère et d'un système de jonctions serrées entre les faces latérales des cellules de Sertoli.

Chacune des deux fonctions testiculaires est sous la dépendance d'une des deux gonadotrophines hypophysaires : $1^{\circ}$ - le contrôle de la fonction endocrine est le fait de la LH dont la cellule cible exclusive est la cellule de Leydig, $2^{\circ}$ - le contrôle de la spermatogénèse est le fait de la FSH dont la cellule cible exclusive est la cellule de Sertoli.

* présenté à la Ire Jounée Scientifique de la S.A.L.F., Tours, 18 juin 1993
Toutefois, la distinction entre le tissu interstitiel à fonction endocrine, contrôlée par la $\mathrm{LH}$ et le tube séminifère à fonction exocrine, contrôlée par la FSH, ne sous-entend pas une indépendance fonctionnelle. Des interrelations entre les différents types cellulaires des 2 compartiments du testicule apparaissent fondamentales dans le fonctionnement harmonieux du testicule. Les cellules de Leydig influencent les fonctions du tube séminifère; la testostérone, à une concentration intra-testiculaire supérieure à la concentration plasmatique, est en effet nécessaire à la spermatogénèse. A l'opposé, nous allons voir que les cellules de Sertoli vont être capables de réguler les fonctions leydigiennes.

\section{MISE EN ÉVIDENCE DU CONTROLE PARACRINE DES FONCTIONS LEYDIGIENNES}

Le concept de la régulation des fonctions des cellules de Leydig par les cellules du tube séminifère voisin, s'est développé à partir de plusieurs types d'observations:

1) La FSH agit indirectement sur la cellule de Leydig : la FSH augmente significativement la sécrétion de testostérone par les testicules de lapin perfusés en présence de concentrations maximales de LH [10]. L'administration de FSH à des rats immatures hypophysectomisés, non seulement stimule la croissance des tubes séminifères mais aussi, induit une hypertrophie des cellules de Leydig [11], une 
augmentation du nombre de récepteurs à la LH et une stimulation des capacités de sécrétion de testostérone en réponse à la $\mathrm{LH}[17,7$, $30,16]$. Par contre, il n'y a pas de stimulation directe de la sécrétion de testostérone par la FSH. Comme les récepteurs à la FSH ne sont présents que sur les cellules de Sertoli, la stimulation des fonctions leydigiennes par la FSH, observée in vivo, nécessite l'intermédiaire des cellules de Sertoli. L'architecture bicompartimentale du testicule laisse supposer que l'effet indirect de la FSH sur les cellules de Leydig est le fait de facteurs diffusant du tubule vers le tissu interstitiel.

2) Les études morphologiques et biologiques portant sur des tubes séminifères isolés ont montré que l'aspect morphologique des cellules de Leydig [4] et la sécrétion de testostérone [18] varient selon le stade du cycle spermatogénétique dans le tubule voisin.

3) Une destruction ou altération spécifique des cellules de la lignée germinale, induites expér:mentalement par divers moyens (irradiation, déficit en vitamine A, ligature des voies efférentes, cryptorchidie, réchauffement) [23], provoquent secondairement des modifications morphologiques et fonctionnelles des cellules de Sertoli et des cellules de Leydig. Lorsque l'altération de la lignée germinale est unilatérale [21] ou locale [1], les modifications secondaires des cellules de Sertoli et des cellules de Leydig sont limitées autour des lésions induites.

Ainsi plusieurs arguments plaident en faveur d'un contrôle local des fonctions des cellules de Leydig par des facteurs paracrines issus du tube séminifère voisin, la production de ces facteurs apparaissant régulable par la FSH et par le stade du cycle spermatogénétique.

\section{EXPÉRIENCES DE COCULTURE DE CELLULES DE LEYDIG ET DE CELLULES DE SERTOLI.}

Des études réalisées in vitro, par culture, en milieu chimiquement déf'pi, de cellules de Leydig, de cellules de Sertoli et des 2 types cellulaires en coculture, ont été conduites en particulier avec des cellules testiculaires de porc immature [22].

La coculture augmente le nombre de récepteurs à LH à la surface des cellules de Leydig et la réponse stéroïdogénique à l'hCG par rapport à celle observée dans la cellule de Leydig cultivée isolément. Un traitement préalable par FSH renforce cette action. Des modifications de l'ultrastructure de la cellule de Leydig avec développement du réticulum endoplasmique et accumulation de gouttelettes lipidiques se produit en coculture [27]. Ces modifications sont similaires à celles observées lors du traitement par FSH d'animaux hypophysectomisés $[17,11]$. On note que de manière symétrique, la coculture augmente les récepteurs à la FSH à la surface des cellules de Sertoli et la sécrétion d'activateur du plasminogène par les cellules de Sertoli en réponse à une stimulation par la FSH. Cet effet est renforcé par un traitement préalable de la coculture par l'hCG. Ces effets ne sont pas retrouvés si les cellules de Leydig ou de Sertoli sont cocultivées avec des cellules non testiculaires.

On observe donc en coculture un effet de stimulation des fonctions différenciées des cellules de Leydig par les cellules de Sertoli traitées par la FSH et une stimulation des fonctions différenciées des cellules de Sertoli par les cellules de Leydig traitées par l'hCG.

\section{NATURE DIFFUSIBLE ET MULTIPLICI- TÉ DES FACTEURS PARACRINES.}

Les études de l'influence sur les cellules de Leydig en culture, des milieux conditionnés de cellules de Sertoli permettent de retrouver un effet stimulant des fonctions des cellules de Leydig si les cellules de Sertoli ont été cultivées en présence d'insuline et de FSH. Par contre si les cellules de Sertoli ont été cultivées en l'absence d'insuline et de FSH, l'effet du milieu conditionné de cellules de Sertoli est plutôt inhibiteur [19]. Ces résultats mon- 
trent d'une part que les phénomènes observés en coculture sont médiés par des facteurs diffusibles et d'autre part que les facteurs paracrines influençant les fonctions différenciées des cellules somatiques du testicule sont vraisemblablement multiples, certains ayant des effets stimulants d'autres inhibiteurs. La production de ces facteurs est influencée par les gonadotrophines.

\section{ROLE DES FACTEURS DE CROISSANCE.}

Deux facteurs de croissance apparaissant médier certains effets des cellules de Sertoli sur les cellules de Leydig ont été plus particulièrement étudiés quant à leur rôle potentiel de facteur paracrine : l'IGF I stimule et le TGF $\beta$ inhibe les fonctions des cellules de Leydig [3] :

\section{1. le TGF $\beta$}

Les cellules de Leydig de porc possèdent des récepteurs spécifiques au TGF $\beta$. Un traitement préalable des cellules de Leydig avec du TGF $\beta$, provoque une inhibition dose dépendante du nombre de récepteurs à $\mathrm{LH} / \mathrm{hCG}$, de la production d'AMPc et de testostérone par la cellule de Leydig, en réponse à une stimulation par hCG. Le milieu conditionné de cellules de Sertoli de porc cultivées en l'absence d'insuline et de FSH contient une substance ayant une activité TGF $\beta$. Ce milieu conditionné a un effet comparable à celui du TGF $\beta$ pur [2]. La présence d'ARN messagers spécifiques dans les cellules de Sertoli est en faveur de la sécrétion de TGF $\beta$ par les cellules de Sertoli. La FSH diminue les ARN messagers du TGF $\beta$. Le TGF $\beta$ pourrait ainsi représenter l'un des facteurs paracrines inhibiteurs des fonctions leydigiennes contenus dans le milieu conditionné des cellules de Sertoli. Le TGF $\beta$ pourrait en outre avoir un effet autocrine puisque des ARN messagers du TGF $\beta$ sont aussi présents dans les cellules de Leydig.

\section{2. l'IGF-I}

Les cellules de Leydig de porc possèdent des récepteurs spécifiques à l'IGF-I. L'IGF-I augmente le nombre de récepteurs à $\mathrm{LH} / \mathrm{hCG}$, la production d'AMPc et de testostérone par la cellule de Leydig, en réponse à une stimulation par hCG $[5,20]$. Les cellules de Sertoli sécrètent un peptide qui a les mêmes propriétés physicochimiques et biologiques que l'IGF-I purifié humain et l'IGF-I obtenu par génie génétique. Sa sécrétion est stimulée par la FSH. L'IGF-I est aussi un facteur autocrine. Les cellules de Leydig de porc sécrètent aussi de l'IGF-I, sa sécrétion est stimulée par l'hCG. L'IGF-I a un rôle trophique sur les fonctions sertoliennes. La coculture de cellules de Leydig et de cellules de Sertoli provoque une stimulation synergique de la sécrétion d'IGF-I. Ainsi, l'IGF-I pourrait correspondre à l'un des facteurs auto-paracrine responsable de l'effet favorable de la coculture sur les fonctions différenciées des cellules testiculaires.

De nombreuses molécules connues pour avoir des fonctions hormonales ou de facteurs de croissance ont été étudiées par de nombreuses équipes pour leur rôle potentiel de facteur paracrine intratesticulaire. On peut citer le GnRH [24], l'arginine-vasopressine [12], l'angiotensine II [13], 1e corticotropin-releasing factor [29], l'interleukine $1 \alpha$ [32], les catécholamines [8], l'epidermal growth factor/TGF- $\alpha$ [31], ou encore l'inhibine et l'activine [9]. Alors que pour beaucoup de ces facteurs il est difficile de préciser leur importance physiologique in vivo en ce qui concerne l'IGF-I, les données obtenues in vitro peuvent être rapprochées d'observations cliniques et d'expérimentations conduites in vivo.

Dans l'espèce humaine, le déficit isolé en $\mathrm{GH}$ est souvent associé à un développement insuffisant du pénis, une puberté retardée et une réponse à l'hCG inférieure à la normale. Le traitement par l'hormone de croissance améliore la maturation pubertaire et la réponse à 
l'hCG $[14,26]$. L'effet de l'hormone de croissance, observé in vivo sur la fonction leydigienne, n'apparaît être qu' indirect, par le biais de l'IGF-I. De même in vivo, le traitement par la GH ou l'IGF-1 de souris déficitaires en GH, provoque une augmentation du nombre de récepteurs à $\mathrm{LH}$ et de la capacité stéroïdogénique des cellules de Leydig [6].

L'IGF-I apparaît donc avoir un rôle central dans la régulation des fonctions testiculaires; l'IGF-I d'origine hépatique sécrété sous l'effet de l'hormone de croissance et l'IGF-I produit localement dans le testicule, sous l'effet des gonadotrophines paraissent conjuguer leurs effets dans la maturation et le maintien des fonctions différenciées des cellules testiculaires.

\section{DANS L'ESPECE HUMAINE.}

Dans le testicule humain adulte, du fait de la difficulté dans l'obtention de tissu testiculaire normal, les études des interrelations paracrines sont moins avancées. En utilisant des testicules de sujets adultes jeunes en état de mort cérébrale récente, prélevés au moment où les reins sont prélevés pour greffe, nous avons pu montrer que la sécrétion de testostérone qui diminue progressivement in vitro -orsque les cellules de Leydig sont cultivées isolément, augmente très nettement lorsque les cellules de Leydig sont cultivées avec des cellules de Sertoli [15]. Comme dans les modèles animaux, les cellules de Sertoli apparaissent capables de favoriser l'expression des fonctions différenciées des cellules de Leydig, même si dans le modèle de porc immature, l'effet des cellules de Sertoli porte surtout sur la réponse des cellules de Leydig à l'hCG, alors que chez l'homme adulte, l'effet de la coculture se manifeste surtout sur la sécrétion basale de testostérone (figure 1).

Ces résultats confirment l'idée, issue d'obser-

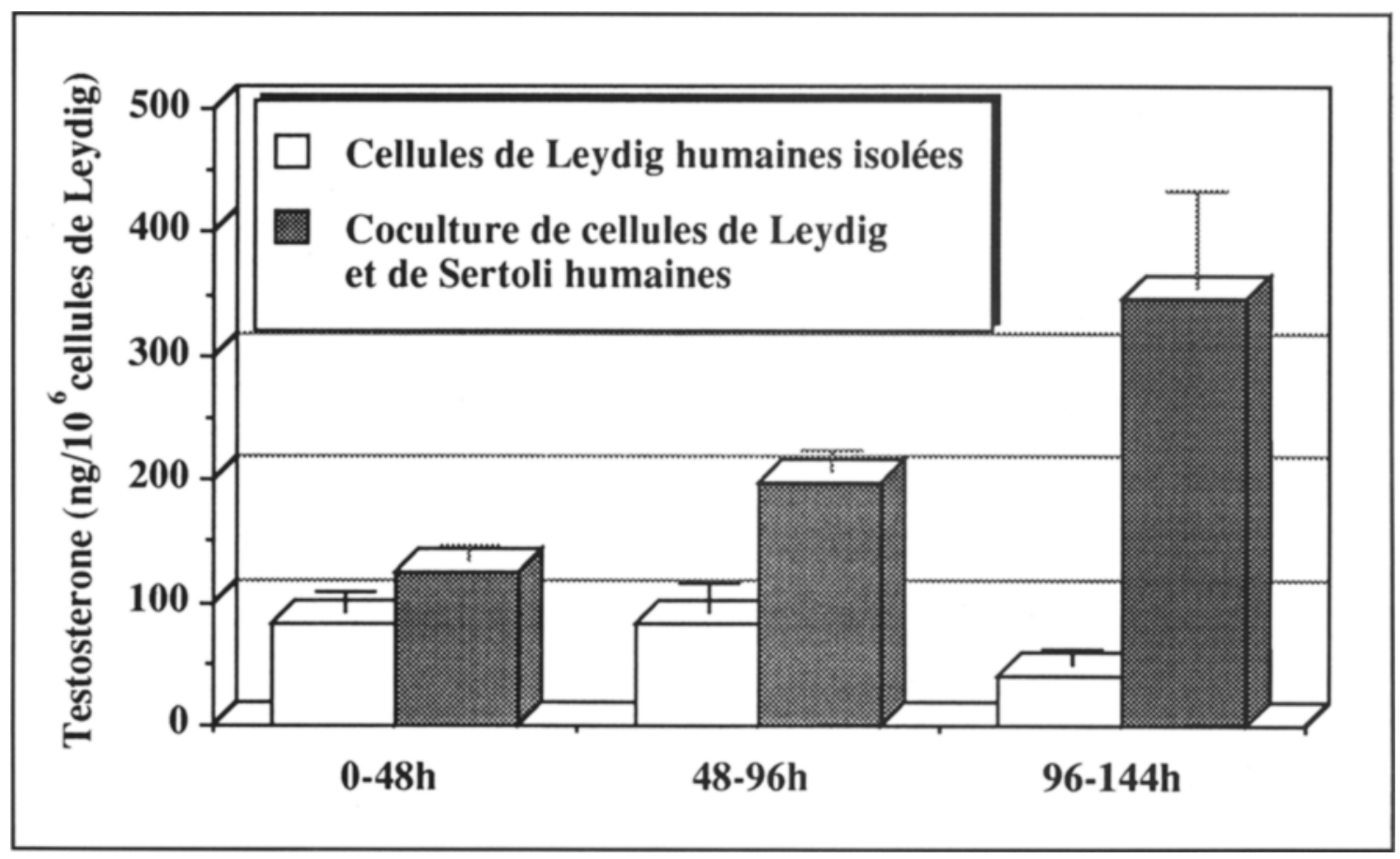

Figure 1. Sécrétion de testostérone par les cellules de Leydig humaines cultivées isolément ou en coculture avec les cellules de Sertoli humaines pendant 3 périodes successives de 48 heures. Les résultats sont la moyenne $\pm S E M$ de 6 cultures indépendantes. Redessiné d'après (15). 
vations cliniques, selon laquelle, chez l'homme comme dans les modèles animaux, le tube séminifère module les fonctions des cellules de Leydig. Il a en effet été rapporté que pendant la puberté chez des enfants présentant une cryptorchidie, la réponse à l'hCG est inversement corrélée au taux de FSH [25]. Par ailleurs, les patients présentant une altération des tubes séminifères ont une testostérone plas. aatique inférieure à la normale malgré des taux de LH élevés [28].

Il apparaît ainsi qu'un fonctionnement normal du testicule nécessite d'une part une stimulation coordonnée des cellules de Leydig par la LH et des cellules de Sertoli par la FSH, ainsi que des interactions entre les divers types cellulaires dans le testicule, mettant en jeu des facteurs de croissance comme facteurs paracrines et autocrines de régulation des fonctions testiculaires.

\section{RÉFÉRENCES}

1. AoKi A, FAwCETT DW. Is there a local feedback from seminiferous tubules affecting activity of the Leydig cell? Biol. Reprod., 1978, 19 : 144-158.

2. Avallet O, Vigier M, Perrard-Sapori MH, Saez JM. Transferming growth factor $\beta$ inhibits Leydig cell functions. Biochem. Biophys. Res. Commun., $1987,146: 575-581$.

3. Avallet O, Vigier M, Chatelain PG, Saez JM. Regulation by growth factors of Leydig cell differentiated functions. J. Steroid Biochem. Molec. Biol., 1991, 40: 453-64.

4. BerGH A. Local differences in Leydig cell morphology in the adult rat testis: evidence for a local control of Leydig cells by adjacent seminiferous tubules. Int. J. Androl., 1982, 5 : 325-330.

5. Bernier M, Chatelain P, Mather JP, Saez JM. Regulation of gonadotropin receptor, gonadotropin responsiveness and cell multiplication by somatomedin-C and insulin in cultured pig Leydig cells. J. Cell. Physiol., 1986,129: 257-263.

6. Chatelain PG, Sanchez P, Saez JM. Growth hormone and insulin-like growth factor I treatment increase testicular luteinizing hormone receptors and steroidogenic responsiveness of growth hormone deficient dwarf mice. Endocrinology, 1991, 128: 1857-1862.
7. Chen YDI, Shaw MJ, Payne AH. Steroid and FSH action on $\mathrm{LH}$ receptors and $\mathrm{LH}$-sensitive testicular responsiveness during sexual maturation of the rat. Mol. Cell. Endocr., 1977, 8 : 291-299.

8. Cooke BA, Golding M, Dix CJ, Hunter MG. Catecholamine stimulation of testosterone production via cyclic AMP in mous Leydig cells in monolayer culture. Mol. Cell. Endocrinol., 1982, 27 : 221-231.

9. Hsueh AJW, Dahl KD, Vaughan J, Tucker E, Rivier J, Bardin CW, VALE W. Heterodimers and homodimers of inhibin have different paracrine action in the modulation of luteinizing hormonestimulated androgen biosynthesis. Proc. Natl. Acad. Sci. USA., 1987, 84 : 5082-5086.

10. Johnson BH, EwING LL. Follicle-stimulationg hormone and the regulation of testosterone secretion in the rabbit testis. Science, 1971, $173: 635-637$.

11. KERR JB, SHARPE RM. Follicle-stimulating hormone induction of leydig cell maturation. Endocrinology, 1985, $116: 2592-2604$.

12. Kasson BG, Adashi EY, Hsueh AJW. Argininevasopressin in the testis : an intragonadal peptide control system. Endocrine Rev., 1985, 7 : 156-168.

13. Khanum A, Dufau ML. Angiotensin II receptors and inhibitory actions in Leydig cells. J. Biol. Chem., 1988, $263: 5070-5074$.

14. Kulin HE, Samoslik E, Santen R, Santner S. The effect of growth hormone on the leydig cell response to chorionic gonadotropin in boys with hypopituitarism. Clin. Endocrinol., $1981 ; 15: 462-472$.

15. Lejeune H, Skalli M, Sanchez P, Avallet $O$, SAEZ JM. (1993). Enhancement of testosterone secretion by normal adult human Leydig cells by co-culture with enriched preparations of normal adult human Sertoli cells. Int. J. Androl., 1993, 16 : 27-34.

16. Moger WH, Murphy PR. Reevaluation of the effect of follicle-stimulating hormone on the steroidogenic capacity of the testis : the effects of neuraminidase-treated FSH preparations. Biol. Reprod., $1982,26: 422-428$.

17. Odell WD, Swerdloff RS. Etiologies of sexual maturation : a model system based on the sexually maturing rat. Rec. Prog. HornL Res., 19767 $32: 245-288$.

18. Parvinen M, Nikula $\mathrm{H}$, Huhtaniemi I. Influence of rat seminiferous tubules on Leydig cell testostérone production in vitro. Mol. Cell. Endocrinol., 1984, $37: 331-336$. 
19. Perrard-Sapori MH, Chatelain PG, Rogemond N, SAEZ JM. Modulation of Leydig cell functions by culture with Sertoli cells of with Sertoli cell conditioned medium : Effects of insulin, somatomedin-C and FSH. Mol. Cell. Endocrinol., 1987, $50: 193-201$.

20. Perrard-Sapori MH., Chatelain PG, Jaillard C, SAEZ JM. Characterization acu gulation of somatomedin-C/Insulin-like growth factor I (Sm-C) receptors on cultured pig Leydig cells. Eur. J. Biochem., 1987, $165:$ 209-214.

21. Risbridger GP, Kerr JB, Peake Ra, De Kretser DM. Assessement of Leydig cell function after bilateral or unilateral efferent duct ligation : further evidence for a local control of Leydig cell function. Endocrinology, 1981,108: 1234-1241.

22. Saez JM, Avallet O, Naville D, Perrard-Sapori MH, Chatelain PG. Sertoli-Leydig cell communications. Ann. N. Y. Acad. Sci., 1989, 564 : 210231.

23. Sharpe RM. Local control of testicular function. QJ1. Exp. Physiol., 1983, 68: 265-282.

24. SHARPE RM. Intratesticular factors controlling testicular function. Biol. Reprod., 1984, 30:29-49.

25. Sizonenko PC, Cuendet A, Paunier L. FSH. I. Evidence for its mediating role on testosterone secretion in cryptorchidism. J. Clin. Endocrinol. Metab., 1973, $37: 68-73$.

26. TANner JM, Whitehouse RH. A note on the bone age at which patients with true isolated growth hormone deiciency enter puberty. J. Clin. Endocrinol. Metab., 1975, $41: 788-790$.

27. Tabone E, Benhamed M, Reventos J, Saez JM. Interaction between immature porcine Leydig and Sertoli cells in vitro. Ultrastructural and biochemical study. Cell. Tiss. Res., 1984, 237 : 357-362.

28. Tsatsoulis A, Whithead E, St. John J, Shalet SM, ROBERTSON WR. The pituitary -Leydig cell axis in men with severe damage to the germinal epithelium. Clin. Endocrinol., 1987, 27 : 683-689.

29. Ulisse S, FabBri A, Dufau ML. Corticotropinreleasing factor receptors and actions in rat Leydig cells. J. Biol. Chem., 1989, $264: 2156-2163$.

30. Van Beurden wmo, Roodnat B, De Jong LH, VAN DER MOLen HJ. Hormonal regulation of LH stimulation of testosterone production in isolated Leydig cells of immature rats; the effect of hypophysectomy, FSH and estradiol-17b. Steroids, $1976,28: 247-259$.

31. Verhoeven G, Cailleau J. Stimulatory effects of epi- dermal growth factor on steroidogenesis in Leydig cells. Mol. Cell. Endrcinol., 1986, 47 : 57-68.

32. Verhoeven G, Cailleau J, Van Damme J, Billiau A. Interleukin-1 stimulates steroidogenesis in cultured rat Leydig cells. Mol. Cell. Endocrinol., 1988, $57: 51-60$. 